Article

\title{
Electrospray Deposition and Magnetic Properties of Prototypical Molecular Magnets
}

\author{
Fabian Paschke 1,*, Philipp Erler ${ }^{1}$, Luca Gragnaniello ${ }^{1}$, Jan Dreiser ${ }^{2}$, \\ Mikhail Fonin 1 ,* \\ 1 Department of Physics, University of Konstanz, Konstanz 78457, Germany \\ 2 Swiss Light Source, Villigen PSI 5232, Switzerland \\ * Correspondence: Fabian Paschke, Email: fabian.paschke@uni.kn; \\ Tel.: +49-7531-88-3985; Mikhail Fonin, Email: mikhail.fonin@uni.kn; \\ Tel.: +49-7531-88-3787.
}

\section{ABSTRACT}

The controlled deposition, characterization and manipulation of single molecule magnets (SMMs) on surfaces is one of the crucial points to be addressed with regard to their possible implementation as functional units in future electronic and spintronic devices. Profound understanding of molecule-molecule and molecule-substrate interactions is required as well as unraveling their effect on the molecular electronic and magnetic properties. Local measurement techniques like scanning tunneling microscopy (STM) together with ensemble-averaging methods like X-ray absorption spectroscopy (XAS) have been proven to set up an appropriate frame to study these materials. The majority of these studies deal with SMMs that exhibit rather simple structures with mostly only one magnetic ion. The situation gets more complicated when it comes to larger polynuclear compounds that can be quite fragile with respect to surface deposition or not easy to organize on surfaces due to their bulky ligand shell. Here, we provide an overview of our results on successful deposition of polynuclear SMMs on functional surfaces by employing the electrospray ion beam deposition method. For two prototypes in the field, $\mathrm{Mn}_{12}$-ac and $\mathrm{Fe}_{4} \mathrm{H}$, we obtain highly ordered submonolayers on functional surfaces and elucidate the electronic coupling to the respective substrates using scanning tunneling spectroscopy (STS). New results for $\mathrm{Mn}_{12}$-ac on

\section{G Open Access}

Received: 29 November 2019

Accepted: 10 January 2020

Published: 16 January 2020

Copyright $₫ 2020$ by the author(s). Licensee Hapres, London, United Kingdom. This is an open access article distributed under the terms and conditions of Creative Commons Attribution 4.0 International License. graphene/Ir(111) and for $\mathrm{Fe}_{4} \mathrm{H}$ on $\mathrm{Au}(111)$ are compared to previous studies on a decoupling graphene layer. X-ray magnetic circular dichroism (XMCD) measurements on submonolayers of uniformly aligned $\mathrm{Fe}_{4}$ molecules on both substrates reveal its robust magnetism, showing magnetic anisotropy values similar to bulk.

KEYWORDS: single-molecule magnet; graphene; $\mathrm{Au}(111)$; scanning tunneling microscopy; electrospray deposition; X-ray absorption spectroscopy 


\section{INTRODUCTION}

The discovery of the first single-molecule magnet (SMM), the mixedvalence cluster $\left[\mathrm{Mn}_{12} \mathrm{O}_{12}\left(\mathrm{O}_{2} \mathrm{CCH}_{3}\right)_{16}\left(\mathrm{H}_{2} \mathrm{O}\right)_{4}\right]\left(\mathrm{Mn}_{12}\right.$-ac) marked the beginning of a new exciting research field at the borderline of chemistry, physics and nanoscience [1-3]. SMMs like $\mathrm{Mn}_{12}$-ac exhibit a large ground-state spin ( $S=10$ for $\mathrm{Mn}_{12}$-ac) combined with a large negative zero-field splitting, leading to a magnetic bistability below a blocking temperature of a few Kelvin [4,5]. These properties promise outstanding functionality in smallscale circuits, making SMMs to one of the most investigated topics in the wide field of molecular electronics and spintronics [6]. From a fundamental point of view SMMs provide a remarkable platform to experimentally observe pure quantum mechanical behavior, such as quantum tunneling of magnetization (QTM) [7], theoretically predicted long before their discovery, superexchange between single spins $[8,9]$ and collective effects on surfaces in the Kondo-regime [10]. Although the $\mathrm{Mn}_{12}$ family of compounds has played a particularly prominent role in SMM research over the first decade after its discovery, a large number of polynuclear complexes showing similar properties have been synthesized and characterized [3]. The main driving force for the synthesis of new compounds is the optimization of SMM properties, like maximizing the blocking temperature (record values up to $60-80 \mathrm{~K}$ in 2017/2018 [11,12]), the anisotropy barrier (record value of $1837 \mathrm{~K}$ [13]) or the ground spin state (record value of $S=60$ [14]), in order to stabilize the magnetic properties when approaching room temperature. Another important research direction is the characterization and manipulation of the electronic and magnetic properties of individual SMMs. Especially the realization of control schemes, which allow to reliably tune the magnetic properties on a single-molecule level, are of particular importance for a possible implementation of these molecules as functional units in future spintronic devices [6,15-17]. Measurements are often performed by contacting the molecules via two electrodes relying on the mechanical break-junction technique [18,19] or electromigration [20,21]. However, the most defined way to access the properties of single molecules is by means of STM and STS, which provide atomic resolution combined with the access to the local electronic and magnetic properties.

The first step to individually address single molecular nanomagnets is their controlled deposition on surfaces. The coverage can range from isolated molecules to full monolayers or even multilayers [8] of stacked but ordered SMMs. Crucial from this point of view is a uniform adsorption geometry that can clearly be identified and is safely reproduced in each experiment. This objective was achieved by using flat molecules with a compact ligand shell [22]. The most investigated class of metal-organic complexes in this regard are paramagnetic, planar transition metal (3d) phthalocyanines [23] and lanthanoid double decker complexes [24]. Combining atomic spin systems together with a well-defined absorption geometry, they offer a perfect model system to study fundamental 
interactions of their spin with the surrounding molecules or the surface, respectively.

The situation is far more complicated when it comes to complex molecular structures that couple together at least two magnetic ions within the core. This naturally includes a considerable enlargement of the ligand shell, making these structures more fragile and thus less appropriate to be adsorbed in a controlled orientation on surfaces which hamper the investigation of polynuclear SMMs. The first attempt by Cornia et al. in 2003 with the prototype complex $\mathrm{Mn}_{12}$ on gold films [25] was followed by a growing amount of STM and STS studies with different derivatives [26-30]. Although revealing detailed information about structural integrity and electronic transport properties, no ordered molecular islands or uniform adsorption geometry could be achieved. Upon application of XAS and XMCD, magnetic properties were investigated and found to deviate from the bulk [31,32]. Deposition of $\mathrm{Mn}_{12}$ on other substrates did not improve the situation [33-35], as well as prefunctionalizing the gold surface with chain-like molecules to suppress interaction with the surface [36]. Frequent problems were the fragmentation of the molecules or the reduction of the oxidation states of the magnetic ions due to the relatively weak stability of these compounds during thermal sublimation. A breakthrough was the implementation of electrospray ion beam deposition (ES-IBD) as gentle method that allows soft-landing of the large $\mathrm{Mn}_{12}$ compounds in form of $\mu \mathrm{m}$-thick films [37] as well as submonolayers [38-40]. Kahle et al. performed inelastic electron tunneling spectroscopy (IETS) of single $\mathrm{Mn}_{12}$ SMMs on a decoupling $h$-BN layer on $\mathrm{Rh}(111)$ and revealed signatures of quantum magnetism, although slightly altered in comparison with the bulk [40]. At the same time first STM images with unambiguous intramolecular resolution allowed to determine the adsorption geometry of individual compounds upon comparison with calculations [40,41].

\section{ELECTROSPRAY ION BEAM DEPOSITION METHOD}

The technique of electrospray ionization allows gentle deposition of complex, fragile and non-volatile molecules on surfaces in an ultra high vacuum (UHV) environment. This is particularly interesting for compounds that are not suitable for thermal evaporation because of considerable fragmentation. The common design using emitter tip, capillary and skimmer was first introduced by Dole and Whitehouse et al. [42,43] and then widely used in mass spectrometry [44-46]. Dissolved molecules are accelerated under ambient pressure by a voltage drop between emitter tip and capillary and towed into vacuum. At the same time this points out one main disadvantage of the method, as only molecules that are stable in solution can be sprayed reliably. The experimental setup we are using to deposit molecular magnets on surfaces is shown in Figure 1. Several differential pumping stages allow a sample pressure down to $10^{-9} \mathrm{mbar}$. Whereas being sufficient for clean solutions, 
the ion beam handling can be improved by introducing quadrupole ion guides, lens systems, a mass spectrometer and retarding voltages [47-49]. The evolution to well-defined molecular beams of high chemical purity combined with surface science techniques then led to the term of preparative mass spectrometry which is reviewed in Rauschenbach et al. [50]. With increasing demand on sample purity, vacuum pressure, mass selectivity and further parameters the size of the experimental setup scales accordingly, from small easy-to-handle UHV chambers to large room-filling machines. As an example Figure 1b shows our experimental setup of compact size without mass selection that can be easily attached to preparation chambers outside the home lab [51].

(a)

(b)
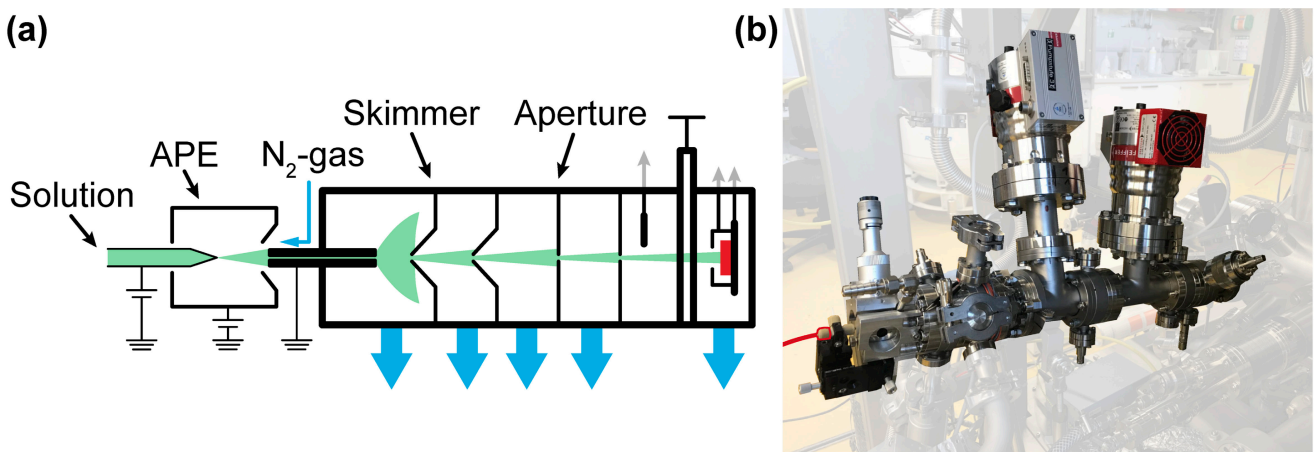

Figure 1. (a) Scheme of the experimental setup used for surface-deposition of molecular magnets discussed in this manuscript. The solvent with molecules (green) is polarized by applying a high voltage ( $\mathrm{kV})$ to the emitter tip. A jet of charged particles is accelerated into the vacuum and can be manipulated by an atmospheric pressure electrode (APE) and counter flow of $\mathrm{N}_{2}$-gas. Blue arrows denote several pumping stages, before the particles land on the sample surface (red). The amount of deposited material is monitored via current measurements (gray arrows). Depending on the purpose, extensions can be realized within the ion beam path, like mass selection and ion guiding. (b) Compact electrospray setup for easy transfer between different UHV preparation chambers.

\section{RESULTS AND DISCUSSION}

In this work we want to emphasize the vital role of the electrospray method with regard to surface deposition of large and fragile molecular magnets. We first focus on the electrospray deposition of prototypical $\mathrm{Mn}_{12}$-ac SMMs on a graphene/Ir(111) surface. Investigation by STM and STS reveals single molecular units on the surface with a defined adsorption geometry and well-separated molecular electronic states, indicating a decent decoupling from the conductive substrate. We then turn to $\mathrm{Fe}_{4} \mathrm{H}$ SMMs, employing the electrospray technique to obtain highlyordered molecular islands on $\mathrm{Au}(111)$ and compare the results with our previous study on graphene/Ir(111). A combined investigation by STM, STS and XAS/XMCD reveals electronic and magnetic properties confirming the robust molecular magnetism of $\mathrm{Fe}_{4}$ complexes upon deposition on substrates. 


\section{$\mathrm{Mn}_{12}$-ac on Graphene/Ir(111)}

$\mathrm{Mn}_{12}$-acetate $\left(\mathrm{Mn}_{12}\right.$-ac) single molecule magnets in a solution of pure methanol were sprayed in situ on a graphene/Ir(111) surface [51]. The apparent height of the molecules on the surface vary between 300-700 pm, depending on tip state and tunneling parameters. Together with unordered molecular islands next to step edges, we also observe periodically arranged molecular units on the bare graphene surface as depicted in Figure 2a. A comparable ordering of $\mathrm{Mn}_{12}$ was not yet observed on other substrates and indicates a decrease of the molecule-substrate interaction on the graphene layer. Lattice parameters of the ordered molecular layer are $a=(1.84 \pm 0.08) \mathrm{nm}, b=(2.23 \pm 0.08) \mathrm{nm}$ and $\varphi=(50 \pm$ $4)^{\circ}$ as shown in Figure 2b. By choosing appropriate tunneling parameters one can simultaneously image $\mathrm{Mn}_{12}$-ac islands and the underlying graphene Moiré pattern, resulting from the atomic lattice mismatch of graphene and the $\operatorname{Ir}(111)$ surface. However, for rather large $\mathrm{Mn}_{12}$ complexes we do not find any correlation between molecular and Moiré unit cell. Intramolecular resolution allows the identification of 10 protrusions per molecule which we assign to the individual acetate ligands. In addition to that we determine an aspect ratio of $(1.38 \pm 0.02)$ for the elliptical shape of the molecules. This agrees well with a $\mathrm{Mn}_{12}$-ac molecule imaged in a slantwise geometry on the surface with the magnetic easy axis being in-plane, see Figure 2c. This arrangement does not reflect the ordering in the crystalline phase but indicates that all magnetic easy axes per island are pointing in the same direction.

To get insight into the local electronic properties of the adsorbed $\mathrm{Mn}_{12^{-}}$ ac complexes we perform scanning tunneling spectroscopy measurements. A normalized $(\mathrm{d} I / \mathrm{d} U) /(\mathrm{I} / U)$ spectrum acquired over the center of a molecule adsorbed at a step edge is shown in Figure 2d. We observe two sharp resonances at negative and positive bias voltage and assign these peaks to tunneling from the highest occupied molecular orbital (HOMO) and into the lowest unoccupied molecular orbital (LUMO) of the $\mathrm{Mn}_{12}$-ac molecule. The sharpness of the resonances reflects a substantial decoupling from the underlying graphene layer. The energy positions of HOMO and LUMO are determined to be $-1.38 \mathrm{eV}$ and $+1.54 \mathrm{eV}$, respectively. This yields a HOMO-LUMO gap of $2.92 \mathrm{eV}$, being comparable to values determined on HOPG [35], Au(111) [51] or obtained in DFT calculations [52]. In addition to the structural integrity this finding underlines the pronounced decoupling from the underlying graphene layer and provides a good basis for further studies on such large polynuclear complexes. 

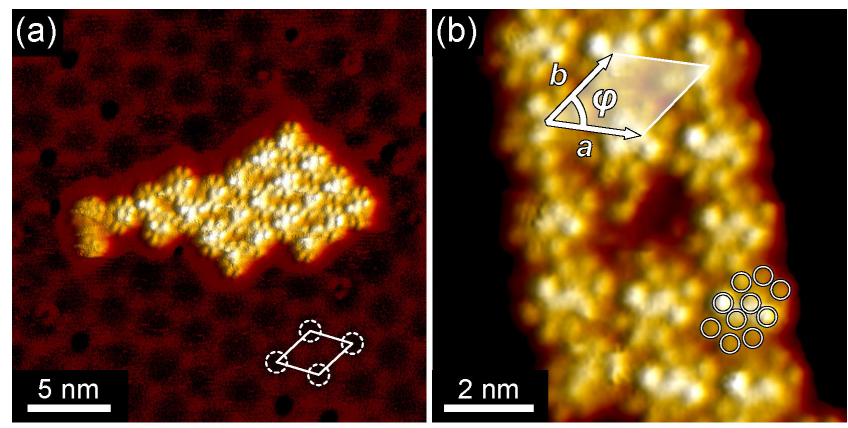

Low Topo (pm) Migh
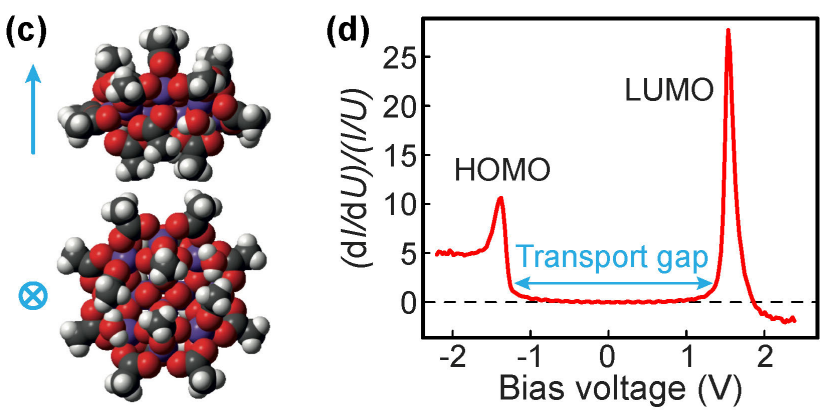

Figure 2. (a,b) STM images of well-ordered $\mathrm{Mn}_{12}$-ac islands on graphene/Ir(111). The substrate's Moiré lattice and molecular unit cell are indicated in white. Intramolecular features are highlighted by white circles. Measurement parameters: $U=+2.3 \mathrm{~V}, I=10 \mathrm{pA}, T=9.9 \mathrm{~K}$. (c) Molecular structure of $\mathrm{Mn}_{12}$-ac, viewed along and perpendicular to the magnetic easy axis (blue arrow). Color code: $\mathrm{Mn}=$ violet, $\mathrm{O}=$ red, $\mathrm{C}=$ gray, $\mathrm{H}=$ white . (d) Normalized $\mathrm{d} I / \mathrm{d} U$ spectrum acquired over the center of $\mathrm{Mn}_{12}$-ac. Distinct peaks mark molecular orbitals. Measurement parameters: $U_{\text {set }}=+2.5 \mathrm{~V}, I_{\text {set }}=50 \mathrm{pA}, T=5.1 \mathrm{~K}$. Normalization was carried out following $(\mathrm{d} I / \mathrm{d} U) /\left((\mathrm{I} / U)^{2}+c^{2}\right)^{1 / 2}$ with $c=5 \mathrm{pS}$ to reflect the density of states most accurately [53]. Data are reproduced from Ref. [51].

\section{Surface Deposition and Properties of $\mathrm{Fe}_{4} \mathrm{H}$}

Difficulties regarding the surface deposition of $\mathrm{Mn}_{12}$ SMMs in a welldefined fashion together with the observation of the loss of SMM properties on surfaces led to the development of new approaches concerning the design of molecular structure and characterization methods [54]. The research was extended to other SMMs, with the observation of QTM by Mannini et al. in a $\left[\mathrm{Fe}_{4}(\mathrm{~L})_{2}(\mathrm{dpm})_{6}\right]$ derivative (with $\mathrm{H}_{3} \mathrm{~L}$ being of the general form $\mathrm{R}-\mathrm{C}\left(\mathrm{CH}_{2} \mathrm{OH}\right)_{3}$ and $\mathrm{Hdpm}=$ dipivaloylmethane) grafted to a gold surface [55,56]. In 2015, magnetic bistability and partially oriented molecules of this so-called $\mathrm{Fe}_{4} \mathrm{SMMs}$ were reported upon adsorption on gold [57]. $\mathrm{Fe}_{4} / \mathrm{Au}(111)$ then served as prototypical system for multi-scale calculation methods [58,59]. However, surface deposition via drop casting or thermal sublimation suffered from degradation due to the molecule's fragile nature [60]. We have recently showed that upon employing the electrospray technique the deposition of intact $\mathrm{Fe}_{4}$ complexes on an insulating $h$-BN/Rh(111) surface can be performed [61]. In order to achieve a preferably flat adsorption configuration we synthesized a new derivative that has the shortest tripodal ligand possible $\left(\mathrm{R}=\mathrm{H}\right.$, called $\mathrm{Fe}_{4} \mathrm{H}$ in the following) as depicted in 
Figure 3a. STM topographic measurements revealed the formation of highly-ordered molecular islands. Upon comparison of STM data to the DFT calculations we could show that all molecules are indeed uniformly lying flat with the plane of their four iron atoms lying parallel to the surface. This particular configuration directly indicates that the easy magnetic axis of every molecule on the surface points in the same direction, that is out-of-plane. These findings are highly relevant for a welldefined contacting of individual molecules as well as the suitability for ensemble measurement methods like XAS and XMCD.

(a)
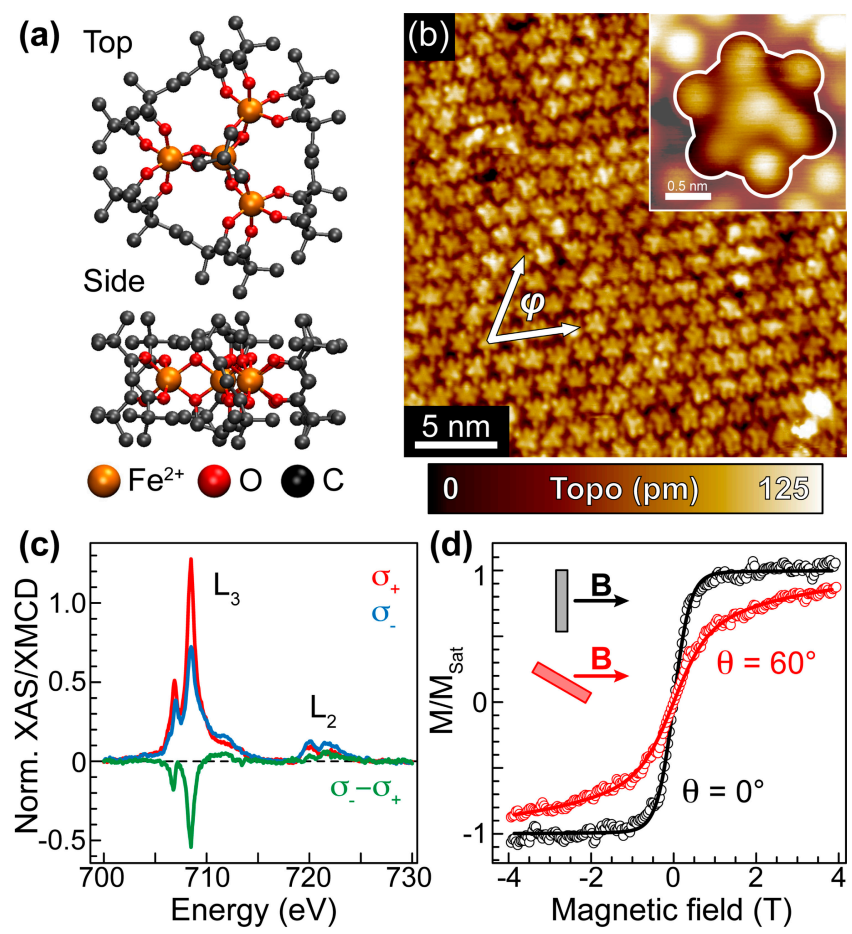

Figure 3. (a) Molecular structure of $\mathrm{Fe}_{4} \mathrm{H}$. $\mathrm{H}$ atoms are omitted for clarity. (b) STM images of $\mathrm{Fe}_{4} \mathrm{H}$ on $\mathrm{Au}(111)$ with the direction of the unit cell vectors sketched in white. Inset: Magnified image of a single molecule with its 3-fold symmetry. Scale bar $0.5 \mathrm{~nm}$, measurement parameters: $U=+2.5 \mathrm{~V}, I=20 \mathrm{pA}, T=4.3 \mathrm{~K}$. (c) XAS/XMCD spectrum of the $\mathrm{Fe}_{4} \mathrm{H}$ submonolayer on $\mathrm{Au}(111)$ at normal incidence of X-ray beam and magnetic field. Measurement parameters: $B=6.8 \mathrm{~T}, T=2.5 \mathrm{~K}$. (d) Magnetization curves obtained at the iron $\mathrm{L}_{3}$ edge for two magnetic field directions. Solid lines represent the fit according to the giant spin model discussed in the text. Measurements were carried out at the X-Treme beamline of the SLS synchrotron at Paul-Scherrer-Institute, Switzerland [62].

\section{$\mathrm{Fe}_{4} \mathrm{H}$ on $\mathrm{Au}(111)$}

Following the intense interest in $\mathrm{Fe}_{4}$ derivatives on gold surfaces, we here present results for the surface deposition of $\mathrm{Fe}_{4} \mathrm{H}$ on $\mathrm{Au}(111)$ using the electrospray method. Figure $3 \mathrm{~b}$ shows an STM topographic image at submonolayer coverage, recorded after cooling down the sample to $4 \mathrm{~K}$. The molecules arrange in an almost hexagonal structure with surface lattice parameters $a=(1.64 \pm 0.03) \mathrm{nm}$ and $b=(1.68 \pm 0.04) \mathrm{nm}$ under an angle of $\varphi=(59.4 \pm 1.9)^{\circ}$. These values are close to the bulk lattice parameters within the crystallographic (001) plane [61]. A zoom on a single 
molecular unit (see inset in Figure 3b) reveals a three-fold symmetry with six protrusions surrounding a central depression or protrusion, depending on bias voltage and tip state. Comparing to the previous results on $\mathrm{Fe}_{4} / h$-BN/Rh(111), we conclude a flat-lying $\mathrm{Fe}_{4} \mathrm{H}$ molecule on the $\mathrm{Au}(111)$ surface with the six lobes corresponding to its outer dpm ligands [61]. At this coverage there are neither preferred adsorption sites nor directional correlations with the herringbone reconstruction of the underlying gold substrate as it is the case for smaller molecules [63,64]. At lower coverage single molecules are found to preferably sitting on elbow sites of the herringbone reconstruction, however they can be easily displaced with the STM tip.

For further investigations on $\mathrm{Fe}_{4} / \mathrm{Au}(111)$ we implemented XAS and XMCD, which have been proven to be powerful tools in order to get deeper insight into electronic states and magnetic properties of adatoms and admolecules on surfaces [54,65]. In the case of $\mathrm{Fe}_{4}$, previous XAS/XMCD studies by Mannini et al. of derivatives grafted to $\mathrm{Au}(111)$ via thiolateterminated aliphatic chains $[32,55,56]$ for the first time demonstrated a system were the striking magnetic properties of SMMs are retained on a conductive surface. The ferrimagnetic spin structure inside $\mathrm{Fe}_{4}$ has been experimentally confirmed by isostructural replacement of the central iron atom to $\mathrm{Fe}_{3} \mathrm{Cr}$ and comparing XMCD spectra of both compounds [66-68]. Derivatives with other tripodal ligands like thioctic side groups $[69,70]$ and short phenyl rings [57] show same spectral characteristics as compared to bulk samples and therefore underline the robust magnetism of the class of $\mathrm{Fe}_{4}$ SMMs. However all of these derivatives exhibit extended tripodal ligands which always result in a certain distribution of adsorption geometries on the gold surface and coupled to this a distribution for the orientation of magnetic easy axes [56,57]. In the following we focus on the flat-lying $\mathrm{Fe}_{4} \mathrm{H}$ derivative with a strictly out-of-plane orientation of the easy magnetic axis.

Figure 3c shows the XAS and derived XMCD spectrum of a submonolayer of $\mathrm{Fe}_{4} \mathrm{H} / \mathrm{Au}(111)$, recorded at the $\mathrm{L}_{2,3}$-edges of iron ( $2 \mathrm{p} \rightarrow 3 \mathrm{~d}$ ) at normal incidence. All spectra are normalized to the maximum of $\left(\sigma_{+}+\sigma_{-}\right) / 2$ around $708.5 \mathrm{eV}$ with $\sigma_{+/-}$referring to left and right circularly polarized light, respectively. Spectral shape and peak magnitudes agree well with previously reported results of other $\mathrm{Fe}_{4}$ derivatives on gold [32,55-57,66,67,69,70]. The splitting and peak ratios of both absorption edges are generally attributed to an $\mathrm{Fe}^{3+}$ oxidation state in an octahedral environment thus indicating the intact core of $\mathrm{Fe}_{4}$. Furthermore it indicates a ferrimagnetic alignment of inner and outer spin magnetic moments at the iron sites that sums up to a total spin ground state of $S=5$ [66]. For a more quantitative analysis sum rules on both XAS and XMCD spectra allow to obtain values for orbital and spin magnetic moments per atom of the respective element [71,72]. These formulae relate the XMCD intensity at both edges to the total absorption spectrum and have been proven to be powerful tools to determine magnetic anisotropies of SMMs 
[73,74]. However one has to be careful with interpretation of observed values due to systematic errors depending on the respective oxidation state, the direction of the magnetization vector and the respective background correction procedure [75]. We estimate the isotropic XAS spectrum as $\sigma_{0}=\left(\sigma_{+}+\sigma_{-}\right) / 2$ following usual approaches [76]. Applying the sum rules to our results we obtain an orbital magnetic moment $\left\langle\mathrm{m}_{\mathrm{L}}\right\rangle=$ $(0.45 \pm 0.08) \mu_{\mathrm{B}} /$ atom and a spin magnetic moment $\left\langle\mathrm{m}_{\mathrm{s}, \text { eff }}\right\rangle=(1.43 \pm 0.23)$ $\mu_{\mathrm{B}} /$ atom. Errors denote standard deviations upon an ensemble of measurements obtained on four different spots on the surface. There is a non-negligible orbital contribution to the total magnetic moment of $~ 30 \%$. Concerning $\left\langle\mathrm{m}_{\mathrm{s} \text {,eff }}\right\rangle$ it was shown that the sum rule underestimates obtained values by $\sim 30 \%$ in $\mathrm{d}^{5}$ systems [75], thus after applying the correction we obtain a mean spin magnetic moment of $\left\langle\mathrm{m}_{\mathrm{s} \text {, corr }}\right\rangle \sim 2.0$ $\mu_{\mathrm{B}} /$ atom.

To obtain information about magnetic anisotropy of the system we track the peak intensity of the XMCD $\mathrm{L}_{3}$ peak $(708.5 \mathrm{eV})$ upon sweeping the magnetic field. In Figure $3 \mathrm{~d}$ we plot the resulting magnetization curve measured under normal $\left(0^{\circ}\right)$ and grazing $\left(60^{\circ}\right)$ incidence. A steeper increase in the magnetization for normal incidence is a clear indication for an out-of-plane magnetic easy axis. This finding is in line with the STM observations of flat-lying molecules as discussed before. The long acquisition time leads to non-negligible X-ray beam damage as detected by a monotonous decrease of the XMCD $\mathrm{L}_{3}$ edge after several hours, therefore the curves are symmetrized with respect to zero field. This does not affect the overall shape of curves as we measure above the blocking temperature in the superparamagnetic regime and thus no opening of hysteresis or QTM can be observed here [55,56]. To quantitatively analyze both curves we use the giant spin hamiltonian $H_{\mathrm{ZFS}}=D S_{\mathrm{z}}{ }^{2}+g \mu_{0} \mu_{\mathrm{B}} \mathbf{S H}$ that describes prototypical SMMs of spin $\mathbf{S}$ with $S_{z}$ being its out-of-plane projection, $D$ the magnetic anisotropy, $g$ the Landé factor and $\mathbf{H}$ the external magnetic field. Assuming $g=2$ and a $S=5$ spin ground state the fitting procedure yields a magnetic anisotropy value $D_{\text {fit }}=-(57 \pm 17) \mu \mathrm{eV}$ at a temperature $T_{\text {fit }}=(2.9$ $\pm 0.3) \mathrm{K}$ and the resulting curves are displayed as solid lines in Figure 3d. The magnetic anisotropy is in very good agreement with its bulk value of $-53 \mu \mathrm{eV}$ [61] and values reported for other $\mathrm{Fe}_{4}$ derivatives [32,56], both on gold and in bulk. We therefore conclude the SMM magnetic properties of $\mathrm{Fe}_{4} \mathrm{H}$ being fully retained upon deposition on the $\mathrm{Au}(111)$ surface.

\section{$\mathrm{Fe}_{4} \mathrm{H}$ on Graphene/Ir(111)}

In order to be able to investigate the magnetism of $\mathrm{Fe}_{4}$ at the molecular scale Gragnaniello et al. investigated the $\mathrm{Fe}_{4} \mathrm{H}$ compound on a graphene monolayer on $\operatorname{Ir}(111)$ [77]. In contrast to the pronounced corrugation of the Moiré pattern of $h$-BN/Rh(111) [61], graphene/Ir(111) offers the advantage to appear almost flat in height and the work function modulation is reduced to a minimum. This is confirmed by our STM measurements where the $\mathrm{Fe}_{4} \mathrm{H}$ molecular magnets indeed exhibit 
exceptionally well-ordered molecular islands, see Figure 4a. The arrangement of the molecules within the islands is highly periodic with an intermolecular distance of (1.6 \pm 1$) \mathrm{nm}$ and the surface unit cell angle of $(58.1 \pm 1.8)^{\circ}$, which are comparable to those observed on gold and in bulk. In contrast to the $\mathrm{Fe}_{4} \mathrm{H} / h$-BN system no correlation is observed between the molecular assembly and the Moiré superstructure of the substrate. Furthermore, no isolated $\mathrm{Fe}_{4} \mathrm{H}$ molecules are found on the surface, confirming a rather weak interaction of $\mathrm{Fe}_{4} \mathrm{H}$ with graphene.
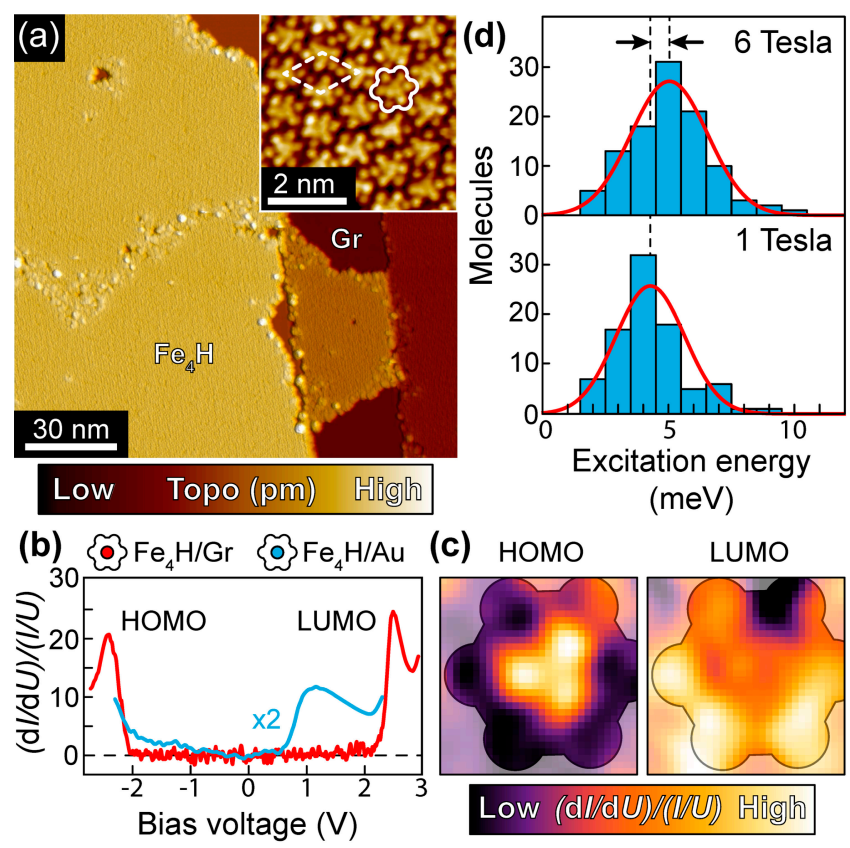

Figure 4. (a) STM image of a submonolayer $\mathrm{Fe}_{4} \mathrm{H}$ on graphene/Ir(111). Inset: High resolution STM image of molecules within the island. The surface unit cell and single molecule shape are highlighted in white. Measurement parameters: $U=+3 \mathrm{~V}, I=10 \mathrm{pA}, T=1.9 \mathrm{~K}$. (b) $(\mathrm{d} I / \mathrm{d} U) /(I / U)$ spectra recorded over the center of $\mathrm{Fe}_{4} \mathrm{H}$ on graphene/Ir(111) (red) and $\mathrm{Au}(111)$ (blue). (c) Spatially resolved molecular orbitals of $\mathrm{Fe}_{4} \mathrm{H}$ on graphene at energies $-2.05 \mathrm{eV}$ (HOMO) and +2.4 eV (LUMO). (d) Statistics of excitation steps observed in IETS of $\mathrm{Fe}_{4} \mathrm{H} /$ graphene/Ir(111). A magnetic field induced shift indicates the magnetic origin of the observed inelastic feature. Results of $\mathrm{Fe}_{4} \mathrm{H} /$ graphene/Ir(111) adapted with permission from Refs. [77,78], Copyright @ $(2017,2019)$ American Chemical Society.

Figure $4 \mathrm{~b}$ shows normalized $(\mathrm{d} I / \mathrm{d} U) /(\mathrm{I} / U)$ spectra acquired over the molecular center. Again resonances at negative and positive bias voltages denote tunneling into the HOMO and LUMO of the molecule. In the case of $\mathrm{Fe}_{4} \mathrm{H} /$ graphene/Ir(111) both HOMO and LUMO orbitals are almost symmetrically located around the Fermi level. The average conductance gap is determined to $(4.5 \pm 1) \mathrm{eV}$, its large size indicating a pronounced electronic decoupling from the substrate. However, a pronounced difference is visible in the spectrum measured on $\mathrm{Fe}_{4} \mathrm{H} / \mathrm{Au}(111)$, which is plotted as blue curve in Figure $4 \mathrm{~b}$. Whereas the intensity at negative bias voltages rises comparably to the result on graphene the LUMO resonance is significantly shifted towards the Fermi level by $\sim(-1.3) \mathrm{eV}$. The decrease of LUMO energy together with the resulting reduction of the transport gap 
to $\sim(2.5 \pm 0.5) \mathrm{eV}$ both indicate an increased coupling to the metal substrate. The STS measurements performed on a grid over a $\mathrm{Fe}_{4} \mathrm{H}$ molecule allows to image the spatial localization of molecular states. Figure $4 \mathrm{c}$ shows the orbitals recorded on $\mathrm{Fe}_{4} \mathrm{H} /$ graphene/Ir(111) at energies corresponding to HOMO and LUMO, respectively. Whereas LUMO is spread over the dpm ligand shell, HOMO is localized over the molecular center, which points at weak coupling to the substrate [61]. In principle this method allows comparison of experimentally observed molecular orbitals with electronic states obtained by DFT calculations, both from the energetic and spatial point of view. However it remains an experimentally challenging task to distinguish between different orbitals as the size of polynuclear SMMs lead to a large number of energy levels lying close together in energy. As the sharpness of orbital resonances in $\mathrm{d} I / \mathrm{d} U$ spectroscopy depends on the molecule-substrate coupling, an appropriate choice of material can therefore enhance the insight in electronic properties of surfacesupported molecules. In our case this is illustrated by the distinction between a conductive gold and a semiconducting graphene surface, with an increased spectral resolution on the latter.

Up to now magnetic properties of $\mathrm{Fe}_{4} \mathrm{H}$ were addressed just by ensemble averaging measurement techniques. On a local scale, inelastic electron tunneling spectroscopy (IETS) has been shown to provide detailed insight into excitation processes of surface-supported vibrational and spin degrees of freedom [79,80]. IETS measurements on artificial atomic structures allowed Hirjibehedin et al. to precisely investigate the interaction between several atomic spin systems [81,82]. As next step the method had been successfully implemented to probe superexchange interactions and magnetic anisotropy of molecular complexes [83,84]. An important advance towards the characterization of polynuclear SMMs was the employment of IETS on polynuclear $\mathrm{Mn}_{12}$ and $\mathrm{Fe}_{4}$ compounds within the tunnel junction $[40,85]$. In these studies the position of excitation steps in $\mathrm{d} I / \mathrm{d} U$ spectra are related to excited spin transitions and allow to determine both the magnetic anisotropy $D$ and the intramolecular exchange interaction parameter $J$. A main drawback in both cases was the bulky structure of the studied molecules leading to an adsorption configuration only roughly determined by STM imaging and thus to an undefined orientation of the magnetic easy axis. With our flat-lying derivative, $\mathrm{Fe}_{4} \mathrm{H}$, we assure a well-defined adsorption configuration as well as well-defined and reproducible orientation of the easy axis. Carefully adjusting tunneling and tip-approach parameters we previously performed IETS measurements on a large number of molecules to obtain reliable statistics, giving the possibility to quantitatively evaluate the impact of the magnetic field [78]. At the same time this procedure captures the variation of step energies that are present within the submonolayer. Resulting histograms are shown in Figure $4 \mathrm{~d}$. The observed excitation energies of $(4.3 \pm 1.4) \mathrm{meV}$ and $(5.0 \pm 1.5) \mathrm{meV}$ in a $1 \mathrm{~T}$ and $6 \mathrm{~T}$ out-of-plane magnetic field allowed us to determine the Fe-Fe exchange interaction $J$ 
based on a Heisenberg and giant-spin model. The obtained $J=(1.5 \pm 4) \mathrm{meV}$ is very close to the value of $1.7 \mathrm{meV}$ measured on $\mathrm{Fe}_{4} \mathrm{H}$ bulk material [61] and is in very good agreement with values reported for other $\mathrm{Fe}_{4}$ derivatives [56,57,66,67,85]. Thus we successfully implement the experimental protocol for IETS measurements on large SMM complexes, giving particularly important insights into the properties of surfacesupported individual nanomagnets. The robust magnetism of $\mathrm{Fe}_{4} \mathrm{H}$ on both gold and graphene however raises the question about the connection to the profound differences of electronic states found in $\mathrm{d} / \mathrm{d} U$ spectroscopy. Thus, further STM studies are needed to address the electronic and magnetic states of individual complexes in order to shed light on details of intramolecular exchange, magnetic anisotropy, as well as the substrateinduced renormalization of electronic states. To this end a joint effort of theoretical modelling and precise local investigations on well-defined systems is needed to reveal the influence of surface deposition on electronic and magnetic properties of large SMMs.

\section{CONCLUSION}

We here review the recent results on the successful deposition of prototypical SMMs $\mathrm{Mn}_{12}$ and $\mathrm{Fe}_{4}$ on surfaces by employing the electrospray deposition technique. STM topographic imaging in both cases revealed mainly intact molecules after adsorption, thus highlighting the potential of electrospray as a well suitable deposition method for larger and rather fragile molecular magnets. The used derivatives $\mathrm{Mn}_{12}$-ac and $\mathrm{Fe}_{4} \mathrm{H}$ arrange in highly-ordered submonolayers on a graphene/ $\operatorname{Ir}(111)$ surface and intramolecular resolution allows us to determine their adsorption configurations. In the case of $\mathrm{Mn}_{12}$-ac all magnetic easy axes are supposed to be co-aligned within each molecular island, thus forming a cone of preferred directions similar to $\mathrm{Fe}_{4}$ with extended tripodal ligands. We present results of $\mathrm{Fe}_{4} \mathrm{H}$ deposited on a $\mathrm{Au}(111)$ surface and observed a similar arrangement to the case on graphene/Ir(111) with a flat adsorption geometry. XAS and XMCD measurements confirmed a bulk-like magnetic anisotropy with all magnetic easy axes pointing in the out-of-plane direction, indicating the robust magnetism of the ligand shell-protected magnetic core of $\mathrm{Fe}_{4}$. For both SMMs we performed $\mathrm{d} I / \mathrm{d} U$ spectroscopy on single molecules that revealed well-separated HOMO and LUMO orbitals and indicate a substantial decoupling from the underlying substrate. However the transport gap of $\mathrm{Fe}_{4} \mathrm{H}$ is significantly reduced by changing from graphene/ $\operatorname{Ir}(111)$ to $\mathrm{Au}(111)$ pointing to a stronger interaction between SMM and the substrate. We also were able to reliably study $\mathrm{Fe}_{4} \mathrm{H}$ nanomagnets on graphene/Ir(111) with IETS, making it a very attractive system for further investigations related to molecular magnetism. This work illustrates how a combination of suitable deposition technique, specific molecular engineering and careful choice of substrate allows to preservethe integrity and study the unique magnetic properties of fragile large, polynuclear molecular magnets. 


\section{AUTHOR CONTRIBUTIONS}

FP, PE, LG and MF designed the study. All authors performed the experiments. FP wrote the manuscript with input from all authors.

\section{CONFLICTS OF INTEREST}

The authors declare that there is no conflict of interest.

\section{FUNDING}

This work was supported by the Deutsche Forschungsgemeinschaft (DFG) through SFB 767. Furthermore the research leading to these results has received funding from the European Union's Horizon 2020 research and innovation programme under grant agreement no. 730872, project CALIPSOplus.

\section{ORCID}

Fabian Paschke: 0000-0002-9710-170X

Luca Gragnaniello: 0000-0003-1150-3941

Jan Dreiser: 0000-0001-7480-1271

Mikhail Fonin: 0000-0003-3015-0045

\section{ACKNOWLEDGEMENTS}

The authors acknowledge V Enenkel, P Schmitt, N Barth, H Brune, S Rusponi, and M Studniarek for useful discussions and experimental assistance.

\section{REFERENCES}

1. Gatteschi A, Caneschi A, Pardi L, Sessoli R. Large Cluster of Metal Ions: The Transition from Molecular to Bulk Magnets. Science. 1994;265:1054-8.

2. Gatteschi D, Sessoli R, Villain J. Molecular Nanomagnets. Oxford (UK): Oxford University Press; 2006.

3. Sieklucka B, Pinkowicz D, editors. Molecular Magnetic Materials: Concepts and Applications. Weinheim (Germany): Wiley-VCH; 2017. ISBN: 978-3-52733963-2.

4. Caneschi A, Gatteschi A, Sessoli R, Barra AL, Brunel L, Guillot M. Alternating Current Susceptibility, High Field Magnetization, and Millimeter Band EPR Evidence for a Ground $S=10$ State in $\left[\mathrm{Mn}_{12} \mathrm{O}_{12}\left(\mathrm{CH}_{3} \mathrm{COO}\right)_{16}\left(\mathrm{H}_{2} \mathrm{O}\right)_{4}\right]$. $2 \mathrm{CH}_{3} \mathrm{COOH} \cdot 4 \mathrm{H}_{2} \mathrm{O}$. J Am Chem Soc. 1991;113:5873-4.

5. Sessoli R, Tsai HL, Schake AR, Wang W, Vincent JB, Folting K, et al. High-Spin Molecules: [ $\left.\mathrm{Mn}_{12} \mathrm{O}_{12}\left(\mathrm{O}_{2} \mathrm{CR}\right)_{16}\left(\mathrm{H}_{2} \mathrm{O}\right)_{4}\right]$. J Am Chem Soc. 1993;115:1804-16.

6. Sanvito S. Molecular Spintronics. Chem Soc Rev. 2011;40:3336-55.

7. Friedman JR, Sarachik MP, Tejada J, Ziolo R. Macroscopic Measurement of Resonant Magnetization Tunneling in High-Spin Molecules. Phys Rev Lett. 1996;76:3830-3. 
8. Chen X, Fu YS, Ji SH, Zhang T, Cheng P, Ma XC, et al. Probing Superexchange Interaction in Molecular Magnets by Spin-Flip Spectroscopy and Microscopy. Phys Rev Lett. 2008;101:197208.

9. Amokrane A, Klyatskaya S, Boero M, Ruben M, Bucher JP. Role of $\pi$-Radicals in the Spin Connectivity of Clusters and Networks of Tb Double-Decker SingleMolecule Magnets. ACS Nano. 2017;11:10750-60.

10. Zhao A, Li Q, Chen L, Xiang H, Wang W, Pan S. Controlling the Kondo Effect of an Adsorbed Magnetic Ion Through its Chemical Bonding. Science. 2005;309:1542-4.

11. Goodwin CAP, Ortu F, Reta D, Chilton NF, Mills DP. Molecular Magnetic Hysteresis at 60 Kelvin in Dysprosocenium. Nature. 2017;548:439-42.

12. Guo FS, Day BM, Chen YC, Tong ML, Mansikkamäki A, Layfield RA. Magnetic Hysteresis up to 80 Kelvin in a Dysprosium Metallocene Single-Molecule Magnet. Science. 2018;326(6421):1400-3. doi: 10.1126/science.aav0652

13. Guo FS, Day BM, Chen YC, Tong ML, Mansikkamäki A, Layfield RA. A Dysprosium Metallocene Single-Molecule Magnet Functioning at the Axial Limit. Angew Chem. 2017;56:11445-9.

14. Baniodeh A, Magnani N, Lan Y, Buth G, Anson CE, Richter J, et al. High Spin Cycles: Topping the Spin Record for a Single Molecule Verging on Quantum Criticality. NPJ Quantum Mat. 2018;3:10.

15. Candini A, Klyatskaya S, Ruben M, Wernsdorfer W, Affronte M. Graphene Spintronic Devices with Molecular Nanomagnets. Nano Lett. 2011;11:2634-9.

16. Urdampilleta M, Nguyen NV, Cleuziou JP, Klyatskaya S, Ruben M, Wernsdorfer W. Molecular Quantum Spintronics: Supramolecular Spin Valves Based on Single-Molecule Magnets and Carbon Nanotubes. Int J Mol Sci. 2011;12:6656-67.

17. Godfrin C, Ferhat A, Ballou R, Klyatskaya S, Ruben M, Wernsdorfer W, et al. Operating Quantum States in Single Magnetic Molecules: Implementation of Grover's Quantum Algorithm. Phys Rev Lett. 2017;119:187702.

18. Reed MA, Zhou C, Muller CJ, Burgin TP, Tour JM. Conductance of a Molecular Junction. Science. 1997;278:252-4.

19. Perrin ML, Verzijl CJO, Martin CA, Shaikh AJ, Eelkema R, van Esch JH, et al. Large Tunable Image-charge Effects in Single-Molecule Junctions. Nat Nano. 2013;8:282-7.

20. Bogani L, Wernsdorfer W. Molecular Spintronics using Single-Molecule Magnets. Nat Mat. 2008;7:179-86.

21. Perrin ML, Burzurí E, van der Zant HSJ. Single-Molecule Transistors. Chem Soc Rev. 2015;44:902.

22. Lei SB, Wang C, Yin SX, Wang HN, Xi F, Liu HW, et al. Surface Stabilized Porphyrin and Phthalocyanine Two-Dimensional Network Connected by Hydrogen Bonds. J Phys Chem B. 2001;105:10838-41.

23. Bartolomé J, Monton C, Schuller IK. Molecular Magnets. Berlin, Heidelberg (Germany): Springer-Verlag; 2014. p. 221-45.

24. Rizzini AL, Krull C, Mugarza A, Balashov T, Nistor C, Piquerel R, et al. Coupling of Single, Double, and Triple-Decker Metal-Phthalocyanine Complexes to Ferromagnetic and Antiferromagnetic Substrates. Surf Sci. 2014;630:361-74. 
25. Cornia A, Fabretti AC, Pacchioni M, Zobbi L, Bonacchi D, Caneschi A, et al. Direct Observation of Single-Molecule Magnets Organized on Gold Surfaces. Angew Chem. 2003;115:1683-6.

26. Coronado E, Forment-Aliaga A, Romero FM, Corradini V, Biagi R, de Renzi V, et al. Isolated $\mathrm{Mn}_{12}$ Single-Molecule Magnets Grafted on Gold Surfaces via Electrostatic Interactions. Inorg Chem. 2005;44:7693-5.

27. Burgert M, Voss S, Herr S, Fonin M, Groth U, Rüdiger U. Single-Molecule Magnets: A New Approach to Investigate the Electronic Structure of $\mathrm{Mn}_{12}$ Molecules by Scanning Tunneling Spectroscopy. J Am Chem Soc. 2007;129:14362-6.

28. Voss S, Fonin M, Rüdiger U, Burgert M, Groth U. Experimental Observation of a Band Gap in Individual $\mathrm{Mn}_{12}$ Molecules on $\mathrm{Au}(111)$. Appl Phys Lett. 2007;90:133104.

29. Voss S, Zander O, Fonin M, Rüdiger U, Burgert M, Groth U. Electronic Transport Properties and Orientation of Individual $\mathrm{Mn}_{12}$ Single-Molecule Magnets. Phys Rev B. 2008;78:155403.

30. Fonin M, Voss S, Herr S, de Loubens G, Kent AD, Burgert M, et al. Influence of the Ligand Shell on the Surface Orientation of $\mathrm{Mn}_{12}$ Single Molecule Magnets. Polyhedron. 2009;28:1977-81.

31. Mannini M, Sainctavit P, Sessoli R, dit Moulin CC, Pineider F, Arrio MA, et al. XAS and XMCD Investigation of $\mathrm{Mn}_{12}$ Monolayers on Gold. Chem Eur J. 2008;14:7530-5.

32. Mannini M, Pineider F, Sainctavit P, Joly L, Fraile-Rodríguez A, Arrio MA, et al. X-Ray Magnetic Circular Dichroism Picks out Single-Molecule Magnets Suitable for Nanodevices. Adv Mat. 2009;21:167-71.

33. Voss S, Fonin M, Zinser F, Burgert M, Groth U, Rüdiger U. A Possible Approach Towards Spin-Polarized Transport through Single Molecule Magnets: $\mathrm{Mn}_{12}$ on $\mathrm{Au}(100) / \mathrm{Fe}(100) / \mathrm{MgO}(100)$. Polyhedron. 2009;28:1606-9.

34. Fleury B, Huc V, Catala L, Jegou P, Baraton L, David C, et al. Orientation of $\mathrm{Mn}_{12}$ Molecular Nanomagnets in Self-Assembled Monolayers. Cryst Eng Comm. 2009;11:2192-7.

35. Meng L, Lin BF, Yang JQ, Sun Y, Dou RF, Ma LX, et al. Detection of the Intrinsic Two Energy Gaps in Individual $\mathrm{Mn}_{12}$ Single Molecule Magnets. Chem Phys Lett. 2012;542:81-4.

36. Moro F, Biagi R, Corradini V, Evangelisti M, Gambardella A, de Renzi V, et al. Electronic and Magnetic Properties of $\mathrm{Mn}_{12}$ Molecular Magnets on Sulfonate and Carboxylic Acid Prefunctionalized Gold Surfaces. J Phys Chem. 2012;116:14936-42.

37. Moroni R, Buzio R, Chincarini A, Valbusa U, de Mongeot FB, Bogani L, et al. Optically Addressable Single Molecule Magnet behavior of Vacuum-sprayed Ultrathin Films. J Mater Chem. 2008;18:109-15.

38. Saywell A, Magnano G, Satterley CJ, Perdigao LMA, Britton AJ, Taleb N, et al. Self-Assembled Aggregates Formed by Single-Molecule Magnets on a Gold Surface. Nat Commun. 2010;1:75. 
39. Saywell A, Britton AJ, Taleb N, Giménez-López MdC, Beton PH, O'Shea JN. Single Molecule Magnets on a Gold Surface: in situ Electrospray Deposition, X-ray Absorption and Photoemission. Nanotechnology. 2011;22:075704.

40. Kahle S, Deng Z, Malinowski N, Tonnoir C, Forment-Aliaga A, Thontasen N, et al. The Quantum Magnetism of Individual Manganese-12-Acetate Molecular Magnets Anchored to Surfaces. Nano Lett. 2012;12:518-21.

41. Sun K, Park K, Xie J, Luo J, Yuan H, Xiong Z, et al. Direct Observation of Molecular Orbitals in an Individual Single-Molecule Magnet $\mathrm{Mn}_{12}$ on $\mathrm{Bi}(111)$. ACS Nano. 2012;7:6825-30.

42. Dole M, Mack LL, Hines RL, Mobley RC, Ferguson LD, Alice MB. Molecular Beams of Macroions. J Chem Phys. 1968;49:2240-9.

43. Whitehouse CM, Dreyer RN, Yamashita M, Fenn JB. Electrospray Interface for Liquid Chromatographs and Mass Spectrometers. Anal Chem. 1985;57:675-9.

44. Fenn JB, Mann M, Meng CK, Wong SF, Whitehouse CM. Electrospray Ionization for Mass Spectrometry of Large Biomolecules. Science. 1989;246:64-71.

45. Kebarle P. A Brief Overview of the Present Status of the Mechanisms Involved in Electrospray Mass Spectrometry. J Mass Spectrom. 2000;35:804-17.

46. Cole RB. Some Tenets Pertaining to Electrospray Ionization Mass Spectrometry. J Mass Spetrom. 2000;35:763-72.

47. Ouyang Z, Takáts Z, Blake TA, Gologan B, Guymon AJ, Wiseman JM, et al. Preparing Protein Microarrays by Soft-Landing of Mass-Selected Ions. Science. 2003;301:1351-4.

48. Rauschenbach S, Stadler FL, Lunedei E, Malinowski N, Koltsov S, Costantini G, et al. Electrospray Ion Beam Deposition of Clusters and Biomolecules. Small. 2006;4:540-7.

49. Rauschenbach S, Vogelgesang R, Malinowski N, Gerlach JW, Benyoucef M, Costantini G, et al. Electrospray Ion Beam Deposition: Soft-Landing and Fragmentation of Functional Molecules at Solid Surfaces. ACS Nano. 2009;3:2901-10.

50. Rauschenbach S, Ternes M, Harnau L, Kern K. Mass Spectrometry as a Preparative Tool for the Surface Science of Large Molecules. Annu Rev Anal Chem. 2016;9:473-98.

51. Erler P. Electronic and Magnetic Properties of Single Molecule Magnets on Surfaces [PhD Thesis]. Konstanz (Germany): University of Konstanz; 2016.

52. Pederson MR, Khanna SN. Magnetic Anisotropy Barrier for Spin Tunneling in $\mathrm{Mn}_{12} \mathrm{O}_{12}$ Molecules. Phys Rev B. 1999;60:9566.

53. Prietsch M, Samsavar A, Ludeke R. Structural and Electronic Properties of the Bi/GaP(110) Interface. Phys Rev B. 1991;43:14.

54. Cornia A, Mannini M, Sainctavit P, Sessoli R. Chemical Strategies and Characterization Tools for the Organization of Single Molecule Magnets on Surfaces. Chem Soc Rev. 2011;40:3076-91.

55. Mannini M, Pineider F, Sainctavit P, Danieli C, Otero E, Sciancalepore C, et al. Magnetic Memory of a Single-Molecule Quantum Magnet Wired to a Gold Surface. Nat Mat. 2009;8:194-7. 
56. Mannini M, Pineider F, Danieli C, Totti F, Sorace L, Sainctavit P, et al. Quantum Tunneling of the Magnetization in a Monolayer of Oriented Single-Molecule Magnets. Nature. 2010;468:417-22.

57. Malavolti L, Lanzilotto V, Ninova S, Poggini L, Cimatti I, Cortigiani B, et al. Magnetic Bistability in a Submonolayer of Sublimated $\mathrm{Fe}_{4}$ Single-Molecule Magnets. Nano Lett. 2015;15:535-41.

58. Garcia GF, Lunghi A, Totti F, Sessoli R. Toward Mesoscale Properties of SelfAssembled Monolayers of SMM on Au(111): An Integrated Ad Hoc FF and DFT Study. J Phys Chem C. 2016;120:14774-81.

59. Garcia GF, Lunghi A, Totti F, Sessoli R. The Disclosure of Mesoscale Behavior of a 3d-SMM Monolayer on Au(111) Through a Multi-level Approach. Nanoscale. 2018;10:4096-104.

60. Lanzilotto V, Malavolti L, Ninova S, Cimatti I, Poggini L, Cortigiani B, et al. The Challenge of Thermal Deposition of Coordination Compounds: Insight into the Case of an $\mathrm{Fe}_{4}$ Single Molecule Magnet. Chem Mater. 2016;28:7693-702.

61. Erler P, Schmitt P, Barth N, Irmler A, Bouvron S, Huhn T, et al. Highly Ordered Surface Self-Assembly of $\mathrm{Fe}_{4}$ Single Molecule Magnets. Nano Lett. 2015;15:4546-52.

62. Piamonteze C, Flechsig U, Rusponi S, Dreiser J, Heidler J, Schmidt M, et al. XTreme Beamline at SLS. X-ray Magnetic Circular and Linear Dichroism at High Field and Low Temperature. J Synch Rad. 2012;19:661-74.

63. Cheng Z, Gao L, Deng ZT, Jiang N, Liu Q, Shi DX, et al. Adsorption Behavior of Iron Phthalocyanine on $\mathrm{Au}(111)$ Surface at Submonolayer Coverage. J Phys Chem C. 2007;111:9240-4.

64. Reecht G, Lotze C, Sysoiev D, Huhn T, Franke KJ. Visualizing the Role of Molecular Orbitals in Charge Transport through Individual Diarylethene Isomers. ACS Nano. 2016;10:10555-62.

65. van der Laan G, Figueroa AI. X-ray Magnetic Circular Dichroism-A Versatile Tool to Study Magnetism. Coord Chem Rev. 2014;277-8:95-129.

66. Mannini M, Tancini E, Sorace L, Sainctavit P, Arrio MA, Qian Y, et al. Spin Structure of Surface-Supported Single-Molecule Magnets from Isomorphous Replacement and X-ray Magnetic Circular Dichroism. Inorg Chem. 2011;50:2911-7.

67. Tancini E, Mannini M, Sainctavit P, Otero E, Sessoli R, Cornia A. On-Surface Magnetometry: The Evaluation of Superexchange Coupling Constants in Surface-Wired Single-Molecule Magnets. Chem Eur J. 2013;19:16902-5.

68. Cornia A, Mannini M, Sessoli R, Gatteschi D. Propeller-Shaped $\mathrm{Fe}_{4}$ and $\mathrm{Fe}_{3} \mathrm{M}$ Molecular Nanomagnets: A Journey from Crystals to Addressable Single Molecules. Eur J Inorg Chem. 2019;2019:552-68.

69. Rodriguez-Douton MJ, Mannini M, Armelao L, Barra AL, Tancini E, Sessoli R. One-step Covalent Grafting of $\mathrm{Fe}_{4}$ Single-Molecule Magnet Monolayers on Gold. Chem Commun. 2011;47:1467-9.

70. Perfetti M, Pineider F, Poggini L, Otero E, Mannini M, Sorace L, et al. Grafting Single Molecule Magnets on Gold Nanoparticles. Small. 2014;10:323-9.

71. Thole BT, Carra P, Sette F, van der Laan G. X-ray Circular Dichroism as a Probe of Orbital Magnetization. Phys Rev Lett. 1992;68:1943-6. 
72. Carra P, Thole BT, Altarelli M, Wang X. X-ray Circular Dichroism and Local Magnetic Fields. Phys Rev Lett. 1993;70:694-7.

73. Stepanow S, Mugarza A, Ceballos G, Moras, P, Cezar JC, Carbone C, et al. Giant Spin and Orbital Moment Anisotropies of a $\mathrm{Cu}$-phthalocyanine Monolayer. Phys Rev B. 2010;82:014405.

74. Stepanow S, Honolka J, Gambardella P, Vitali L, Abdurakhmanova N, Tseng T, et al. Spin and Orbital Magnetic Moment Anisotropies of Monodispersed Bis(Phthalocyanato)Terbium on a Copper Surface. J Am Chem Soc. 2010;312:11900-1.

75. Piamonteze C, Miedema P, de Groot FMF. Accuracy of the Spin Sum Rule in XMCD for the Transition-Metal L Edges from Manganese to Copper. Phys Rev B. 2009;80:184410.

76. Chen CT, Idzerda YU, Lin HJ, Smith NV, Meigs G, Chaban E, et al. Experimental Confirmation of the X-ray Magnetic Circular Dichroism Sum Rules for Iron and Cobalt. Phys Rev Lett. 1995;75:152-5.

77. Gragnaniello L, Paschke F, Erler P, Schmitt P, Barth N, Simon S, et al. Uniaxial 2D Superlattice of $\mathrm{Fe}_{4}$ Molecular Magnets on Graphene. Nano Lett. 2017;17:7177-82.

78. Paschke F, Erler P, Enenkel V, Gragnaniello L, Fonin M. Bulk-Like Magnetic Signature of Individual $\mathrm{Fe}_{4} \mathrm{H}$ Molecular Magnets on Graphene. ACS Nano. 2019;13:780-5.

79. Stipe BC, Rezaei MA, Ho W. Single-Molecule Vibrational Spectroscopy and Microscopy. Science. 1998;280:1732-5.

80. Heinrich AJ, Gupta JA, Lutz CP, Eigler DM. Single-Atom Spin-Flip Spectroscopy. Science. 2004;306:466-9.

81. Hirjibehedin CF, Lutz CP, Heinrich AJ. Spin Coupling in Engineered Atomic Structures. Science. 2006;312:1021-3.

82. Hirjibehedin CF, Lin CY, Otte AF, Ternes M, Lutz CP, Jones BA, et al. Large Magnetic Anisotropy of a Single Atomic Spin Embedded in a Surface Molecular Network. Science. 2007;317:1199-203.

83. Chen X, Fu YS, Ji SH, Zhang T, Cheng P, Ma XC. Probing Superexchange Interaction in Molecular Magnets by Spin-Flip Spectroscopy and Microscopy. Phys Rev Lett. 2008;101:197208.

84. Tsukahara N, Noto K, Ohara M, Shiraki S, Takagi N, Takata Y, et al. AdsorptionInduced Switching of Magnetic Anisotropy in a Single Iron(II) Phthalocyanine Molecule on an Oxidized Cu(110) Surface. Phys Rev Lett. 2009;102:167203.

85. Burgess JA, Malavolti L, Lanzilotto V, Mannini M, Yan S, Ninova S, et al. Magnetic Fingerprint of Individual $\mathrm{Fe}_{4}$ Molecular Magnets under Compression by a Scanning Tunnelling Microscope. Nat Commun. 2015;6:8216.

How to cite this article:

Paschke F, Erler P, Gragnaniello L, Dreiser J, Fonin M. Electrospray Deposition and Magnetic Properties of Prototypical Molecular Magnets. Quantum Mater Res. 2020;1:e200002. https://doi.org/10.20900/qmr20200002 